\title{
Pruebas de reforestación de mangle en una ciénaga costera semiárida de Yucatán, México
}

\author{
Mangrove reforestation tests in a semiarid coastal swamp \\ of Yucatán, México
}

\author{
José Luis Febles-Patrón1, Jorge Novelo López1 \\ y Eduardo Batllori Sampedro1
}

\begin{abstract}
RESUMEN
Como parte del Programa de Restauración Ambiental y Fortalecimiento al Desarrollo Regional en la microcuenca costera de Chabihau, Yucatán, México (2004-2006), se evaluó la sobrevivencia y el crecimiento de plántulas de Rhizophora mangle L. (mangle rojo) y Avicennia germinans (L.) L. (mangle negro), a través de diferentes pruebas de reforestación. Con las nuevas condiciones que se registran en la ciénaga, después de la construcción de puentes en la carretera costera, la sobrevivencia está regulada por el nivel de inundación, mientras que la salinidad del agua controla el crecimiento de las plántulas de manglar. El mangle rojo presentó una mayor sobrevivencia bajo condiciones de mayor inundación y un mayor desarrollo con baja salinidad intersticial (3,28 ups $\pm 0,32$ ups), mientras que el mangle negro presentó una mayor sobrevivencia en áreas menos inundadas y un mayor desarrollo con más alta salinidad $(45,5$ ups $\pm 0,50$ ups). La construcción de camas de sedimento para controlar el grado de inundación demostró ser una técnica útil para favorecer la sobrevivencia de mangle negro, mientras que el desazolve de manantiales y la disminución de la salinidad del agua, aseguran un mejor desarrollo de las plántulas de ambas especies. Se obtuvo una mayor sobrevivencia del mangle rojo producido en el vivero que de la siembra directa. La menor profundidad y mayor salinidad del agua registrados en la ciénaga en 2006, considerado como un año menos lluvioso, pueden estar relacionadas con una mayor repoblación natural de mangle negro. Para el diseño de programas de reforestación se debe contar con un monitoreo de parámetros hidrológicos superficiales y del suelo (particularmente nivel de inundación y salinidad), que permita la selección de las especies y los métodos adecuados para asegurar la mayor sobrevivencia y el mejor desarrollo de las plantas.
\end{abstract}

PALABRAS CLAVE:

Avicennia germinans, Chabihau, reforestación, restauración ambiental, Rhizophora mangle.

\begin{abstract}
Rhizophora mangle L. (red mangrove) and Avicennia germinans (L.) L. (black mangrove) seedling survival and growth were evaluated in reforestation trials as part of an environmental restoration and regional development program in the Chabihau coastal microbasin, Yucatan, Mexico. Building of road bridges over inlets modified swamp conditions by increasing tide reflux and reducing salinity extremes. Mangrove seedling survival is now regulated by flood level and growth by salinity. Red mangrove survival was highest in deeper areas and with higher development with lower soil salinity (3,28 psu $\pm 0,32 \mathrm{psu}$ ), while black mangrove survival was higher in shallower areas and development was highest with higher soil salinity (45,5 psu $\pm 0,50 \mathrm{psu})$. Building of sediment beds to control degree
\end{abstract}


of flooding aided in increasing black mangrove survival, and clearing of springs to reduce salinity ensured proper growth. Survival was higher for nurseryraised red mangrove seedlings than for directly planted red mangrove seedlings. High black mangrove natural incorporation was probably favored by the lower water depth and higher salinity in the swamp during 2006, a low rainfall year. Reforestation programs need to include monitoring of surface and soil hydrological parameters (especially flood level and salinity) to aid in the selection of optimum species and methods for higher seedling survival and improved growth.

KEY WORDS:

Avicennia germinans, Chabihau, reforestation, environmental restoration, Rhizophora mangle-

\section{INTRODUCCIÓN}

Los principales impactos de origen natural y antropogénico que afectan los humedales costeros de Yucatán están relacionados con: 1) la construcción de caminos, 2) la construcción de puertos, 3) el crecimiento de la población, 4) la intrusión de agua marina a través de aperturas en la duna costera, 5) la sedimentación de manantiales y 6) los huracanes. En este aspecto, Batllori et al. (1999) encontraron una pérdida de vegetación de manglar de 1,3 km²/año, entre 1948 y 1991, en los humedales costeros del noroeste de Yucatán, debido principalmente al cambio en el régimen hidrológico y a la hipersalinización. Los manglares son dependientes de la incorporación de plántulas para su mantenimiento (Craighead, 1971; Tomlinson, 1994); por lo tanto, el establecimiento y la sobrevivencia de las plántulas afectan directamente los patrones de distribución y abundancia (McKee, 1995a y b). Ante el cambio climático global, en las próximas décadas se prevé un incremento en el nivel del mar y de la intensidad $y$ frecuencia de los huracanes, lo cual aumentará la vulnerabilidad de los humedales y del desarrollo costero (Yánez-
Arancibia et al., 1998; Batllori et al., 2006). Por lo anterior, es necesario fortalecer las acciones de monitoreo para evaluar las tendencias ambientales que permitan el manejo de los recursos naturales.

Los ecosistemas costeros en Yucatán tienen características particulares, debido a su origen cárstico y a la ausencia de ríos superficiales. Son sistemas generados por la dinámica de las corrientes marinas que forman una isla de barrera o duna costera y los sistemas lagunares, abiertos o cerrados, y que por los aportes de agua dulce subterránea a través de manantiales, adquieren un carácter salobre en algunos sitios y condiciones hipersalinas en otros. En la microcuenca de Chabihau existen tres localidades urbanas, San Crisanto, Chabihau y Santa Clara, conectadas por una red carretera, tanto en la costa como hacia el interior, modificando los flujos de agua y el transporte de sedimentos. Entre las carreteras que seccionan a la laguna con dirección norte-sur están la que une a San Crisanto con Sinanché, otra que conduce de Chabihau a Yobaín, y la carretera de Santa Clara a Dzidzantún. Este ecosistema es muy vulnerable al impacto de huracanes. El huracán Gilberto en 1988 y posteriormente el huracán Isidoro en septiembre de 2002, han ocasionado la ruptura de la barra arenosa, conectando el mar con la laguna en el área oriental de la localidad de Chabihau, cambiando de un régimen estacional a uno dominado por la marea. Esta situación ha traído beneficios sociales y económicos, como el aumento en la pesca de autoconsumo en la laguna, principalmente de camarón en la época de invierno.

En el año 2003 se construyeron dos puentes carreteros en las bocas abiertas por el huracán Isidoro, uno de $24 \mathrm{~m}$ de ancho en el margen oriental de la zona 
urbana de la localidad de Chabihau y otro más de $12 \mathrm{~m}$ de ancho, hacia Santa Clara. Posterior al huracán y la construcción de puentes en el año 2003 en Chabihau y El Porvenir, se presentaron nuevas tendencias en el sistema hidrológico. En el caso de Chabihau, se registraron condiciones de menor salinidad en la ciénaga, cambiando de un sistema hipersalino a otro euhalino, donde la salinidad promedio del agua superficial bajó de 44 ups a 33 ups (las ups o "unidades prácticas de salinidad" son el coeficiente entre la conductividad eléctrica de la solución bajo estudio y la conductividad de una solución de $\mathrm{KCl}$ al $0,1 \%$; y estiman la concentración de sales equivalente, en partes por mil, en una solución estandarizada de cloruro de potasio), con mayor recambio y flujo del agua, correspondiendo también con años más lluviosos (2002-2004; Batllori y Febles, 2007a y b).

En 2003 inició el programa de restauración ambiental y fortalecimiento al desarrollo regional en la microcuenca costera de Chabihau, Yucatán, con el apoyo del North American Wetlands Conservation Council (NAWCC), para desarrollar obras de restauración ambiental a través de la capacitación de mujeres y hombres de la comunidad de Chabihau. De esta manera, se realizó el desazolve de manantiales, la construcción de un vivero de mangle y pruebas de reforestación de manglar en la ciénaga.

\section{OBJETIVOS}

Como parte del programa de restauración ambiental y fortalecimiento al desarrollo regional en la microcuenca costera de Chabihau, Yucatán, México, se evalúa la sobrevivencia y el crecimiento de plántulas de Rhizophora mangle L. (mangle rojo) y Avicennia germinans (L.) L. (mangle negro), a través de diferentes pruebas de reforestación realizadas en la ciénaga de Chabihau.

\section{DESCRIPCIÓN DEL SITIO DE ESTUDIO}

El sistema de humedales costeros de Chabihau se extiende por $17 \mathrm{~km}$, desde la localidad de San Crisanto hasta Santa Clara, y abarca una superficie aproximada de 4500 ha, de las cuales 3400 ha son ocupadas por ciénaga con régimen de marea (1 149 ha) y vegetación de manglar (2 251 ha), además de 1100 ha de selva baja inundable y sabana (Figura 1). Estos humedales se protegen por una barra arenosa con dunas costeras que apenas sobrepasan las 300 ha. De acuerdo con Teutli (2004), las especies de manglar presentes en la zona son $R$. mangle, Laguncularia racemosa y $A$. germinans, con una mayor dominancia de $R$. mangle $(55 \%$ a $62 \%$, lado $\mathrm{E}$ y $\mathrm{O}$ de la carretera). De acuerdo con la misma autora, el lado Este de la ciénaga presenta un mayor desarrollo del manglar con un área basal de $3,83 \mathrm{~m}^{2} / \mathrm{ha}$ $\left(0,40 \mathrm{~m}^{2} /\right.$ ha del lado Oeste $)$, una densidad de $2400 \mathrm{ind} / \mathrm{ha}$ (360 ind/ha del lado Oeste) y una altura promedio de 3,16 $\mathrm{m}(1,89 \mathrm{~m}$ del lado Oeste). Presenta un clima cálido seco del tipo Bso(h')(x')i, de acuerdo con la clasificación de Köppen modificada por García (1988), con temperatura media anual de $26 \circ$. La precipitación anual es de $600 \mathrm{~mm}$, mientras que la evaporación supera el valor de $1800 \mathrm{~mm}$ anuales. La temporada de lluvias inicia en mayo con los meses más lluviosos en junio y septiembre. Existe la presencia de canícula en julio y agosto, y a partir de octubre, la precipitación disminuye, siendo los meses de febrero, marzo y abril los menos húmedos.

El manglar está constituido por los cuerpos de agua superficial permanentes o temporales que conforman la ciénaga y 


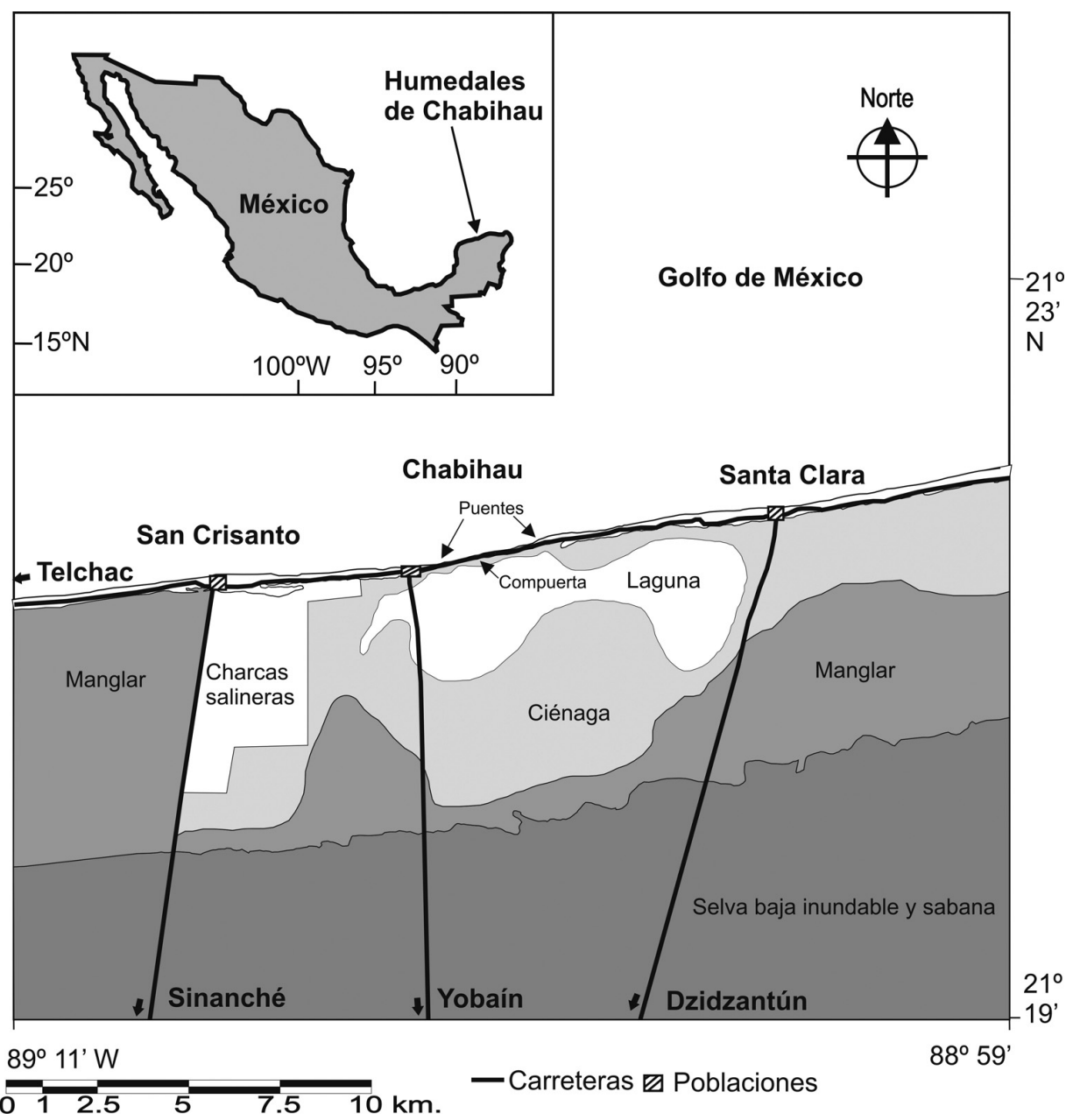

Figura 1. Localización de los humedales costeros de Chabihau, Yucatán.

los demás ambientes inundables. Estos humedales se alimentan de los afloramientos del acuífero subterráneo, de la precipitación pluvial y de las aguas marinas que se filtran o desbordan a través de la barra costera arenosa. Existe un acuífero subterráneo, el cual se mantiene confinado y separado de los humedales por una capa impermeable denominada caliche. En aquellos sitios donde la capa confinante se rompe, el agua aflora a manera de manantiales que representan puntos de descarga del acuífero continental (Perry et al., 1989). En la ciénaga de Chabihau existen numerosos afloramientos de este tipo, con un alto grado de azolvamiento y escaso flujo de agua hacia la ciénaga. Esta situación junto con las condiciones climáticas imperantes en la zona, permiten el predominio de condiciones más salinas en el agua y suelo de la ciénaga. Los suelos denominados solonchac se localizan en la franja de terrenos bajos y pantanosos de las 
ciénagas que se extienden a lo largo de la costa de Yucatán. La mayoría muestran efectos de gleyzación (hidromorfismo), lo que los identifica como solonchac gléyico. Este suelo aluvial es rico en sales solubles y con un rango variable de profundidad a la roca calcárea de $0 \mathrm{~cm}$ a $50 \mathrm{~cm}$. Presenta valores de $\mathrm{pH}$ entre 8 y 9 , rico en calcio y magnesio y relativamente bajo en fósforo (Duch, 1988).

\section{METODOLOGÍA}

En el vivero construido por el grupo de mujeres de Chabihau "Flor de mangle", se prepararon 10000 bolsas de plástico con sedimento de la ciénaga en las que se colocaron 5000 propágulos de mangle rojo y 5000 de mangle negro bajo condiciones de sombra (con techo de palma y sombra durante las horas de mayor insolación). Se regaron dos veces por semana con agua dulce (menos de 5 ups) durante un periodo de cuatro meses (agosto-diciembre de 2004). Se registró una fuerte mortalidad de mangle negro en el vivero, posiblemente asociado con el manejo, por lo que se decidió aumentar la producción de mangle rojo. Posteriormente, en diciembre de 2004 las plantas fueron trasplantadas en los alrededores de tres manantiales localizados en la ciénaga de Chabihau (Alfonsina, Victoria y El Cambio) (Figura 2).

Estos manantiales fueron desazolvados previamente y se construyó un bordo alrededor de ellos con el sedimento extraído, evitando la entrada de agua más salada de la ciénaga. Se excavó un pequeño canal $(0,70 \mathrm{~m}$ de largo, $0,80 \mathrm{~m}$ de ancho y $0,50 \mathrm{~m}$ de profundidad) con un vertedero en los bordos, para permitir el flujo de agua del manantial hacia la ciénaga, en ciertas condiciones de marea. En el área adyacente al manantial Alfonsina, se delimitó

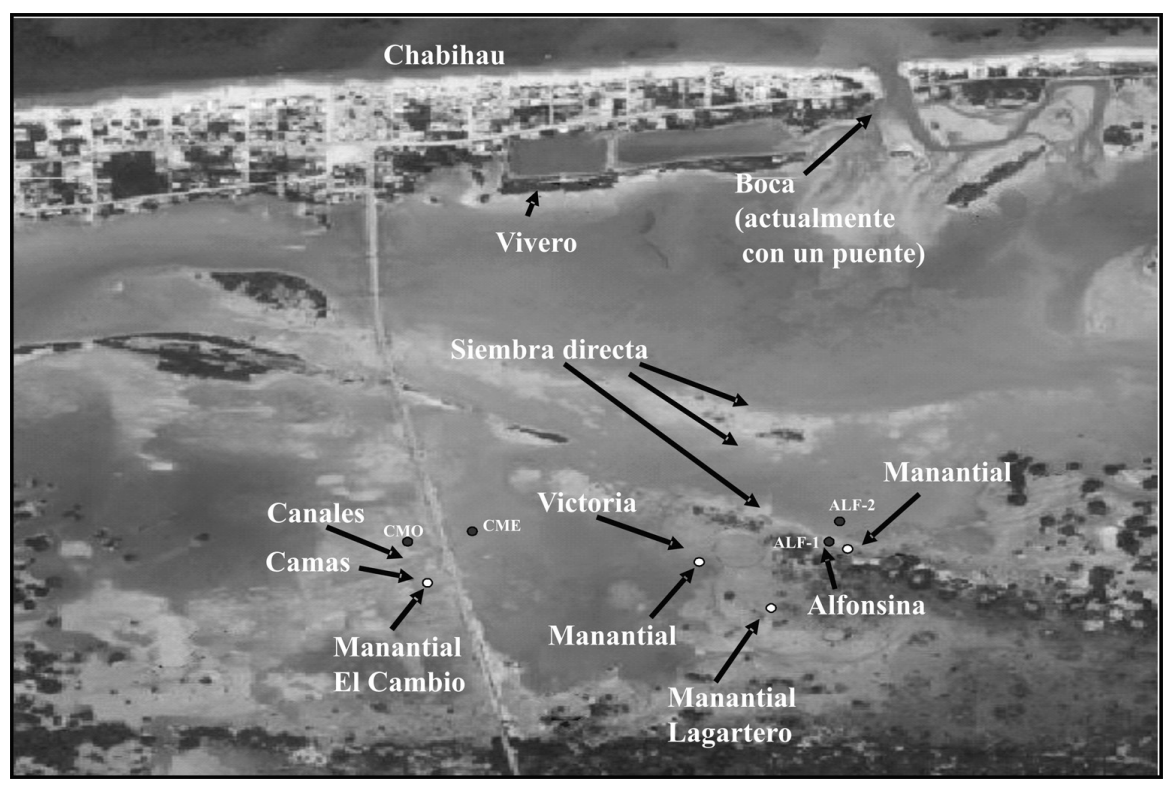

Figura 2. Localización esquemática de la zona de reforestación y sitios cercanos del monitoreo hidrológico en Chabihau, Yucatán. 
una zona con un bordo de madera $(50 \mathrm{~m}$ de largo, 0,15 $\mathrm{m}$ de ancho y $0,75 \mathrm{~m}$ de profundidad) y tela sintética (polietileno de alta densidad), para inducir la acumulación de sedimento y evitar la entrada de agua marina. Por otra parte, para controlar el nivel de inundación se construyeron 17 camas triangulares de sedimento en los alrededores del manantial El Cambio ( $8 \mathrm{~m}$ de longitud a ambos lados y $5 \mathrm{~m}$ en la base), con una elevación aproximada de $25 \mathrm{~cm}$ del suelo. Con la intención de permitir el flujo de agua del manantial El Cambio a la ciénaga, se excavaron $80 \mathrm{~m}$ de canales, de $20 \mathrm{~cm}$ de profundidad y $40 \mathrm{~cm}$ de ancho, aproximadamente; no obstante, estos canales se obstruyeron con sedimento durante el siguiente año.

\section{Tratamientos de trasplante para mangle rojo y negro}

1. Alfonsina: plántulas de vivero en ciénaga, con afluencia de manantial y menor influencia de agua marina.

2. Victoria: plántulas de vivero en ciénaga, con afluencia de manantial y mayor influencia de agua marina.

3. El Cambio: plántulas de vivero en camas de sedimento alrededor de un manantial.

4. Canales: plántulas de vivero en ciénaga, a los lados de canales de drenaje, con menor afluencia de manantiales y mayor influencia de agua marina.

5. Siembra directa: propágulos de mangle rojo en ciénaga (septiembre 2005), en las zonas adyacentes de parches de manglar, con el nivel del suelo más elevado y mayor influencia de agua marina.

Todos los tratamientos fueron bajo condiciones de insolación directa.
En la tabla 1 se presenta la cantidad de plántulas en los diferentes sitios, por especie, procedentes del vivero (cuatro meses de crecimiento) o de la siembra directa. Para conocer la sobrevivencia y el crecimiento de las plántulas se realizó un monitoreo en época de secas (agosto) y en época de inundación (diciembre) durante dos años (diciembre 2004diciembre 2006), que incluyó el número de individuos vivos, la altura y el número de hojas y de ramas por individuo (10\% de la muestra). Se anotó también el número de raíces adventicias, además de la presencia de flores o frutos en los diferentes momentos. El primer tiempo corresponde a los valores en vivero.

De forma complementaria se utilizaron los datos del monitoreo hidrológico de 2002 a 2006 que se han realizado en varios sitios de la ciénaga y manantiales (después del huracán Isidoro). Este monitoreo se realizó con una frecuencia irregular a través del año, en meses diferentes al muestreo biológico, pero se usa como referencia para caracterizar las condiciones hidrológicas en sitios cercanos a las áreas de reforestación. De esta manera, se relaciona el sitio Alf-1 con Alfonsina, la ciénaga media Este (CME) con Victoria, la ciénaga media Oeste (CMO) con El Cambio y Canales, y Alf-2 con la zona de la siembra directa (Figura 2). Se instalaron balizas de madera para medir la profundidad del agua y con un multianalizador YSI Modelo 85 se registró la salinidad (intervalo de 0 ups a 80 ups, $\pm 0,1$ ups), la temperatura (intervalo de $-5 \circ \mathrm{C}$ a $65 \circ \mathrm{C}, \pm$ $0,1 \circ \mathrm{C}$ ) y el oxígeno disuelto (intervalo de $0 \mathrm{mg} / \mathrm{L}$ a $20 \mathrm{mg} / \mathrm{L}, \pm 0,3 \mathrm{mg} / \mathrm{L})$. Con un analizador $\mathrm{YSI}$ pH100 se midió el $\mathrm{pH}$ (intervalo de -2 a $16 \pm 0,01$ ) y el potencial REDOX (intervalo de $-1999 \mathrm{mV}$ a 1250 $\mathrm{mV} \pm 1 \mathrm{mV}$ ). En algunos sitios (Alf-1, CMO y Alf-2) se instalaron tubos de PVC en el sedimento para el monitoreo de los parámetros hidrológicos intersticiales. No 
Tabla 1.- Distribución y densidad de plántulas por sitio y especie en diciembre de 2004.

\begin{tabular}{llll}
\hline Mangle negro & $\begin{array}{l}\text { Cantidad } \\
\text { ind/m² }\end{array}$ & Superficie reforestada (ha) & Densidad \\
\hline Victoria & 230 & 0,76 & 0,030 \\
Alfonsina & 171 & 0,16 & 0,106 \\
El Cambio & 280 & 0,29 & 0,096 \\
Canales & 15 & 0,72 & 0,002 \\
Subtotal & 696 & & Densidad \\
\hline Mangle rojo & Cantidad & Superficie reforestada (ha) & \\
& & & 0,397 \\
ind/m2 & & & 0,75 \\
Victoria & 3,022 & 0,76 & 0,714 \\
Alfonsina & 1,200 & 0,16 & 0,034 \\
El Cambio & 1,500 & 0,21 & 0,192 \\
Canales & 246 & 0,72 & \\
Siembra directa & 3,602 & 1,87 & \\
Subtotal & 9,550 & & \\
Total de plántulas & 10,246 & & \\
& & & \\
\hline
\end{tabular}

se instalaron tubos de PVC en todos los sitios debido a la dureza de la roca presente a poca profundidad del suelo. La Comisión Nacional del Agua (CONAGUA) proporcionó la información climatológica para la estación de Telchac, población costera localizada $14 \mathrm{~km}$ al poniente de Chabihau.

\section{Análisis de la información}

Los datos del número de individuos vivos, por especie y por sitio, para cada fecha de muestreo, además de los valores de altura $(\mathrm{cm})$, número de hojas, de ramas y de raíces, fueron tabulados en Excel y posteriormente en el programa Statistica v. 6 se obtuvieron los estadísticos descriptivos de tendencia central y de dispersión (promedio y error estándar).

Con los valores de altura se realizaron pruebas de Kruskal-Wallis (SPSS v.14) para identificar diferencias temporales en cada sitio y para cada especie, diferencias espaciales en cada fecha de muestreo y para cada especie, además de diferencias entre especies, en cada sitio y para cada fecha de muestreo. Este análisis también fue apoyado con la confección de gráficos en el programa Statistica v. 6.

Los valores de los parámetros hidrológicos también fueron tabulados en hoja de cálculo Excel y en Statistica v. 6 para la obtención de los estadísticos descriptivos. Se obtuvieron los valores para cada sitio de toda la serie y por temporada de secas (marzo-agosto) y de inundación (septiembre-febrero). En el programa SPSS $v .14$ se realizaron pruebas de Kruskal-Wallis para identificar las diferencias espaciales, considerando toda la serie de valores de cada parámetro hidrológico, además de las diferencias temporales en cada sitio para cada parámetro hidrológico. 


\section{RESULTADOS}

\section{Régimen de precipitación pluvial}

La precipitación pluvial registrada en Telchac Puerto mostró una característica estacionalidad, con una marcada época de lluvias, desde mayo a octubre, la presencia de una canícula en pleno verano, y una temporada más seca de noviembre a abril, principalmente. El año 2004 presentó la mayor precipitación con un total de $944,8 \mathrm{~mm}$ (los meses de febrero, mayo, julio y septiembre fueron los más lluviosos). En 2005 la precipitación total fue de $779 \mathrm{~mm}$, con la mayor lluvia concentrada en septiembre y octubre. Finalmente, en 2006 se registró la menor precipitación total anual de $316,4 \mathrm{~mm}$, aunque los meses de marzo y agosto fueron más lluviosos que en los años anteriores (Figura 3).

\section{Sobrevivencia}

La sobrevivencia de propágulos de mangle negro en el vivero fue baja, posiblemente debido al manejo, de manera que solamente se logró producir $14 \%$ de la cantidad programada. Para compensar la baja sobrevivencia del mangle negro se aumentó un $20 \%$ del número de propágulos de mangle rojo, llegando a una producción de 5968 individuos. El total de producción de plantas en el vivero fue de 6664 de ambas especies.

Durante el primer año de crecimiento (diciembre 2004-2005), el mangle negro de Victoria había desaparecido $(0 \%$ de sobrevivencia), mientras que en Alfonsina el mangle rojo presentó una sobrevivencia del $16 \%$. En El Cambio el mangle negro presentó la mayor sobrevivencia con $45,7 \%$, mientras que el mangle rojo en Victoria con $29,5 \%$ (Figura 4). En la siembra directa de propágulos de mangle rojo, se registró una sobrevivencia de $52,2 \%$ de septiembre a diciembre de 2005, mientras que en agosto 2006 (once meses después) bajó hasta $14,2 \%$.

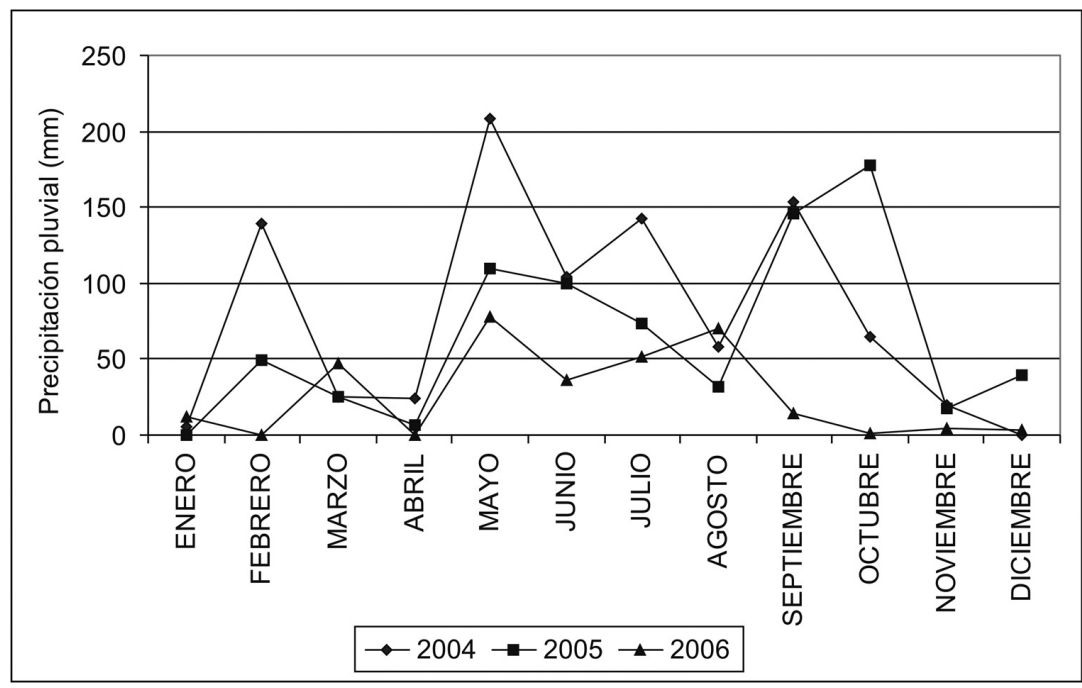

Figura 3. Régimen de lluvia en Telchac Puerto de 2004 a 2006 (CONAGUA, 2006). 

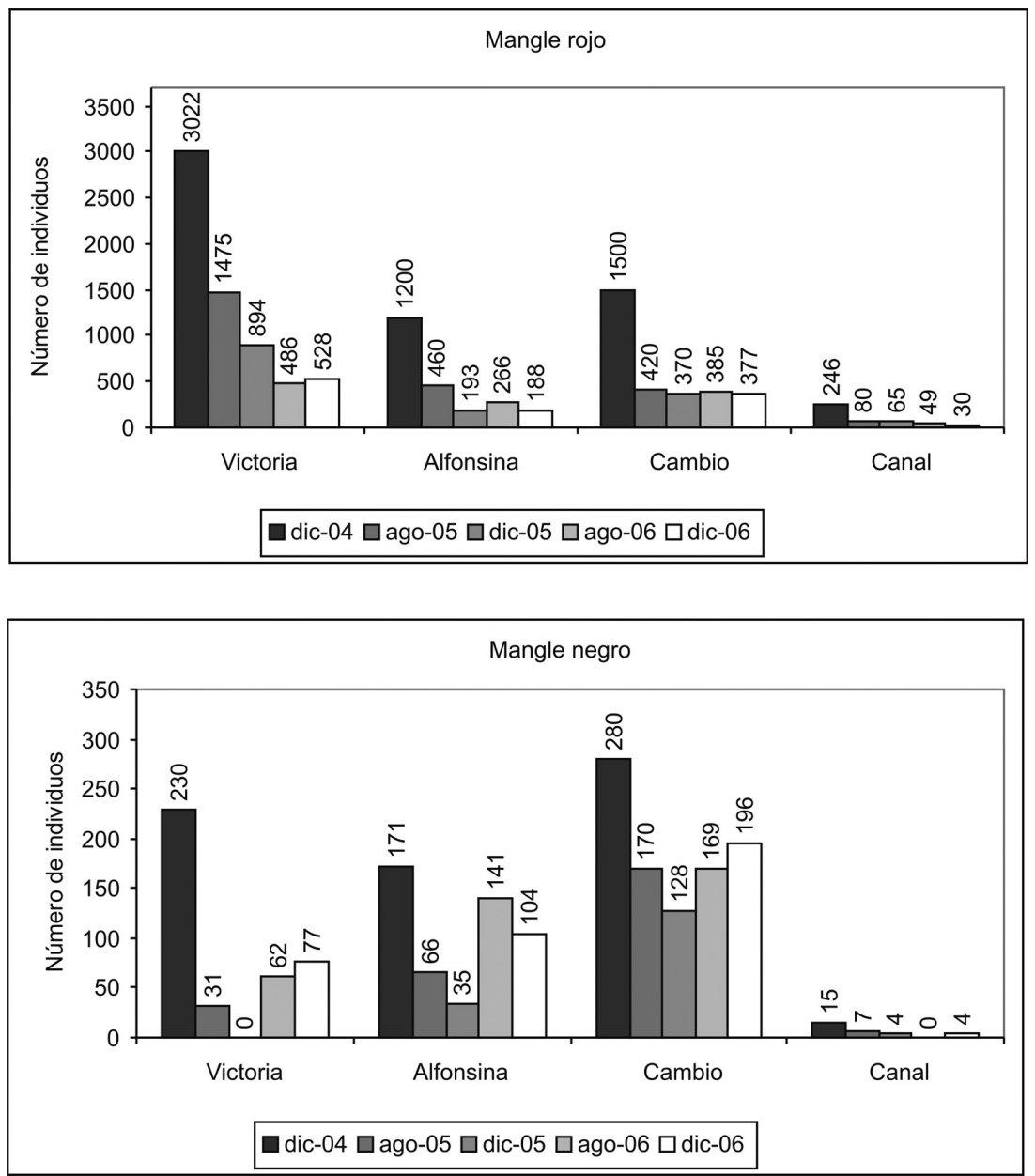

Figura 4. Sobrevivencia de mangle rojo y negro en los sitios de reforestación.

En agosto 2006 se registró un fenómeno de incorporación natural en la mayoría de los sitios. En Victoria se contaron 62 individuos nuevos de mangle negro $(26,9 \%$ del número de plántulas trasplantadas inicialmente). La mayor repoblación natural de mangle negro se registró en Alfonsina $(82,4 \%$ con relación al número inicial de plántulas trasplantadas). En la zona de los canales, adyacentes a $\mathrm{El}$ Cambio, la mortalidad de mangle negro fue total. En la zona de la siembra directa se observó el mismo proceso de incorporación natural que en los otros sitios, con 819 individuos de mangle negro en agosto de 2006; no obstante, para diciembre 2006 había una sobrevivencia de $60,2 \%$ de individuos de mangle negro. Debido a que los individuos no fueron marcados, en cada muestreo fueron contabilizados todos los que se encontraban en cada sitio, incluyendo los incorporados de forma natural, por lo que en la figura 4 se incluye el total de individuos 
presentes en cada momento. Este proceso de repoblación natural se observó también en el último muestreo de diciembre de 2006, dos años después de iniciar la reforestación; no obstante, fue mayor para el mangle negro que para el rojo.

\section{Crecimiento de las plantas}

Desde agosto 2005 y hasta diciembre 2006 (480 días), la mayor altura promedio de las plantas de mangle rojo se registró en Alfonsina, siguiendo en Victoria y en El Cambio. El crecimiento neto en altura fue de $33,4 \mathrm{~cm}$ en Alfonsina, de $27,7 \mathrm{~cm}$ en Victoria y de $26,9 \mathrm{~cm}$ en El Cambio. En Alfonsina se registró también el mayor número promedio de hojas (en diciembre 2006), de ramas y de raíces aéreas, mientras que la zona de los canales presentó el menor desarrollo con un crecimiento neto en altura de $23,7 \mathrm{~cm}$ (Tabla 2). En todos los sitios, las primeras ramas se registraron desde agosto 2005 (240 días), con un mayor desarrollo un año después (agosto 2006, 605 días), cuando se registraron las primeras raíces adventicias, excepto en los canales. Adicionalmente, Alfonsina fue el único sitio donde se observó floración a los 605

Tabla 2. Parámetros biológicos en el mangle rojo de la zona de reforestación de Chabihau.

\begin{tabular}{lccccccccc}
\hline & \multicolumn{3}{c}{ Altura $(\mathrm{cm})$} & \multicolumn{2}{c}{ Hojas } & \multicolumn{2}{c}{ Ramas } & \multicolumn{2}{c}{ Raíces } \\
\hline Sitio & Fecha & Media & EE & Media & EE & Media & EE & Media & EE \\
\hline Vivero & Dic-04 & 15,26 & 0,26 & 1,74 & 0,08 & 0,00 & 0,00 & 0,00 & 0,00 \\
\hline \multirow{2}{*}{ Alfonsina } & Ago-05 & 26,13 & 0,74 & 9,98 & 0,82 & 1,00 & 0,16 & 0,00 & 0,00 \\
& Dic-05 & 30,79 & 0,60 & 10,28 & 0,73 & 0,90 & 0,11 & 0,00 & 0,00 \\
& Ago-06 & 46,31 & 2,35 & 66,15 & 8,32 & 7,27 & 1,10 & 2,08 & 0,44 \\
& Dic-06 & 48,70 & 1,19 & 76,51 & 5,75 & 6,29 & 0,46 & 2,77 & 0,32 \\
\hline \multirow{2}{*}{ Victoria } & Ago-05 & 21,69 & 0,40 & 3,61 & 0,23 & 0,08 & 0,02 & 0,00 & 0,00 \\
& Dic-05 & 29,17 & 0,70 & 5,13 & 0,35 & 0,11 & 0,05 & 0,00 & 0,00 \\
& Ago-06 & 38,26 & 0,94 & 27,12 & 1,94 & 4,56 & 0,98 & 0,42 & 0,17 \\
& Dic-06 & 43,01 & 1,24 & 33,64 & 2,74 & 4,15 & 0,41 & 0,55 & 0,20 \\
\hline \multirow{2}{*}{ Camas } & Ago-05 & 16,24 & 0,75 & 4,60 & 0,33 & 0,16 & 0,08 & 0,00 & 0,00 \\
& Dic-05 & 29,55 & 0,45 & 7,58 & 0,41 & 0,31 & 0,06 & 0,00 & 0,00 \\
& Ago-06 & 38,04 & 1,57 & 33,63 & 3,98 & 3,98 & 0,55 & 0,33 & 0,13 \\
& Dic-06 & 42,17 & 1,47 & 41,18 & 4,65 & 5,16 & 0,63 & 0,98 & 0,24 \\
\hline \multirow{2}{*}{ directa } & Ago-06 & 34,36 & 0,49 & 18,89 & 0,88 & 0,36 & 0,09 & 0,00 & 0,00 \\
& Dic-06 & 35,77 & 0,42 & 22,85 & 0,86 & 2,46 & 0,10 & 0,06 & 0,02 \\
& & & & & & & & & \\
\hline \multirow{2}{*}{ Canales } & Ago-05 & 16,00 & 2,53 & 4,83 & 0,95 & 0,17 & 0,17 & 0,00 & 0,00 \\
& Dic-05 & 30,94 & 0,99 & 5,03 & 0,40 & 0,09 & 0,05 & 0,00 & 0,00 \\
& Ago-06 & 31,00 & 2,74 & 19,00 & 3,99 & 2,00 & 0,84 & 0,00 & 0,00 \\
& Dic-06 & 39,00 & 3,79 & 27,33 & 11,86 & 3,00 & 1,53 & 0,00 & 0,00 \\
\hline
\end{tabular}


días (agosto 2006) y fructificación a los 730 días (diciembre 2006).

Hasta agosto 2006 (605 días) el mangle negro presentó la mayor altura en Alfonsina, seguido de El Cambio. En diciembre 2006 (730 días), El Cambio presentó la mayor altura promedio, junto con Alfonsina, mientras que en Canales se registró la menor altura. La tasa de crecimiento neto fue de $41,9 \mathrm{~cm}$ en $\mathrm{El}$ Cambio y de $38,3 \mathrm{~cm}$ en Alfonsina. En Victoria, la tasa de mortalidad registrada en diciembre de 2005 (365 días) fue de $100 \%$; no obstante, la incorporación natural registrada a partir de agosto 2006
(605 días) presentó un considerable desarrollo en altura, similar al registrado en El Cambio, en esa misma fecha. Nuevamente, en Canales se observó un rápido crecimiento de agosto a diciembre de 2005, aunque un año después (agosto 2006) la mortalidad alcanzó también el 100\%. En diciembre de 2006 se registró un proceso de repoblación natural en los canales, con una tasa neta de crecimiento de $32 \mathrm{~cm}$. En El Cambio se registró el mayor número promedio de hojas y ramas, seguido de Alfonsina y de Victoria, mientras que en Alfonsina se registró el mayor número de raíces adventicias (Tabla 3).

Tabla 3. Parámetros biológicos en el mangle negro de la zona de reforestación de Chabihau.

\begin{tabular}{|c|c|c|c|c|c|c|c|c|c|}
\hline \multirow[b]{2}{*}{ Sitio } & \multirow[b]{2}{*}{ Fecha } & \multicolumn{2}{|c|}{ Altura $(\mathrm{cm})$} & \multicolumn{2}{|c|}{ Hojas } & \multicolumn{2}{|c|}{ Ramas } & \multicolumn{2}{|c|}{ Raíces } \\
\hline & & Media & EE & Media & $\mathrm{EE}$ & Media & $\overline{E E}$ & Media & EE \\
\hline Vivero & Dic-04 & 8,26 & 0,36 & 3,04 & 0,18 & 0,00 & 0,00 & 0,00 & 0,00 \\
\hline \multirow[t]{4}{*}{ Alfonsina } & Ago-05 & 30,20 & 0,93 & 13,14 & 1,12 & 0,48 & 0,13 & 0,00 & 0,00 \\
\hline & Dic-05 & 35,92 & 1,28 & 10,27 & 0,90 & 0,20 & 0,11 & 0,00 & 0,00 \\
\hline & Ago-06 & 42,64 & 3,07 & 33,00 & 6,55 & 2,79 & 0,81 & 2,29 & 0,78 \\
\hline & Dic-06 & 46,65 & 2,53 & 45,12 & 6,30 & 3,06 & 0,46 & 8,53 & 1,63 \\
\hline \multirow[t]{3}{*}{ Victoria } & Ago-05 & 23,23 & 1,27 & 7,52 & 0,89 & 0,23 & 0,11 & 0,00 & 0,00 \\
\hline & Ago-06 & 40,00 & 2,29 & 25,50 & 2,29 & 2,10 & 0,38 & 0,10 & 0,10 \\
\hline & Dic-06 & 44,18 & 2,45 & 49,00 & 10,45 & 4,25 & 1,51 & 7,25 & 1,97 \\
\hline \multirow{4}{*}{$\begin{array}{l}\text { Camas } \\
\text { (El Cambio) }\end{array}$} & Ago-05 & 29,84 & 0,91 & 18,66 & 1,82 & 1,56 & 0,26 & 0,00 & 0,00 \\
\hline & Dic-05 & 30,07 & 1,22 & 20,62 & 1,97 & 1,13 & 0,20 & 0,00 & 0,00 \\
\hline & Ago-06 & 40,00 & 2,29 & 74,64 & 7,73 & 9,05 & 1,05 & 1,95 & 0,49 \\
\hline & Dic-06 & 50,22 & 3,48 & 142,26 & 23,98 & 11,78 & 1,51 & 6,22 & 1,13 \\
\hline \multirow[t]{3}{*}{ Canales } & Ago-05 & 28,14 & 3,11 & 10,86 & 1,96 & 0,29 & 0,29 & 0,00 & 0,00 \\
\hline & Dic-05 & 33,50 & 4,77 & 6,25 & 1,75 & 0,00 & 0,00 & 0,00 & 0,00 \\
\hline & Dic-06 & 32,00 & 5,00 & 16,00 & 4,00 & 0,00 & 0,00 & 0,00 & 0,00 \\
\hline \multicolumn{10}{|c|}{ Repoblamiento } \\
\hline \multirow[t]{2}{*}{ Natural * } & Ago-06 & 34,43 & 1,40 & 15,03 & 1,93 & 0,53 & 0,16 & 0,59 & 0,21 \\
\hline & Dic-06 & 32,66 & 0,97 & 10,82 & 0,99 & 0,20 & 0,07 & 0,16 & 0,11 \\
\hline
\end{tabular}

* en la zona de siembra directa 
En la zona de la siembra directa se registró un aumento en la altura promedio de las plántulas de mangle rojo desde diciembre 2005 hasta diciembre 2006, con una tasa de crecimiento neto de 14,2 $\mathrm{cm}$. Se registraron diferencias significativas en la altura en todos los sitios y en ambas especies a través del tiempo (figuras 5, 6, 7 y 8; $p=0,001$ ). En la mayoría de los casos las diferencias fueron principalmente entre el primer y el segundo año de crecimiento. En términos espaciales, el mangle rojo presentó diferencias entre sitios en agosto $\left(X^{2}=13,385\right.$, $p=0,004) \quad y$ diciembre de 2006 $\left(X^{2}=16,775, p=0,001\right.$, segundo año de crecimiento), mientras que el mangle negro únicamente presentó diferencias en agosto $2005\left(X^{2}=18,065, p=0,001\right)$ y diciembre $2005\left(X^{2}=10,524, p=0,005\right.$, primer año de crecimiento). No hubo diferencias para el mangle negro en agosto $\left(X^{2}=0,552, p=0,759\right)$ y diciembre 2006 $\left(X^{2}=3,292, \quad p=0,349\right.$, segundo año de crecimiento). En todos los sitios se encontraron diferencias entre especies únicamente en el primer año de crecimiento $(p=0,001$ a 0,012$)$.

\section{Monitoreo hidrológico}

Los manantiales Alfonsina y El Cambio presentaron la más baja salinidad promedio del agua superficial, de 3,30 ups $(E E= \pm 1,04)$ y 6,9 ups $(E E= \pm 1,67)$ respectivamente, mientras que la ciénaga media Este y Oeste (CME y CMO), así como el sitio Alf-2, presentaron la más alta salinidad promedio con 39,3 ups $(E E= \pm 2,02), 35,4$ ups $(E E= \pm 2,31)$ y 32,7 ups $(E E= \pm 2,10)$, respectivamente. El manantial Victoria, junto con el sitio Alf-1, presentaron salinidades intermedias (21,9 ups, $E E= \pm 2,15$ y 15,7 ups, $E E= \pm 3,21$, respectivamente). La salinidad máxima registrada fue de 70 ups en la ciénaga media Este y la mínima de 1,1 ups en el manantial Alfonsina. En Alf-1 la salinidad intersticial promedio fue de 3,28 ups $(E E= \pm 0,32)$, mientras que en Alf- 2 fue de 34,6 ups $(E E= \pm 1,67)$ y en la ciénaga media Oeste fue de 45,5 ups $(E E= \pm 0,50)$. El valor máximo fue de 4,7 ups en Alf-1, 48,1 ups en Alf-2 y 46 ups en la ciénaga media Oeste.

La profundidad promedio fue de 5,8 cm $(E E= \pm 3,19)$ en Alf-1, hasta 48,6 ups $(E E= \pm 2,8)$ en el manantial Alfonsina; no obstante, en las áreas de reforestación no sobrepasó los $19 \mathrm{~cm}(\mathrm{EE}= \pm 1,71)$ y 10,2 $(E E= \pm 2,15)$ de profundidad en la ciénaga media Este y Oeste, respectivamente. El oxígeno y el $\mathrm{pH}$, junto con la temperatura, fueron los parámetros hidrológicos con menor variación y con mayor homogeneidad. La temperatura máxima promedio del agua fue de 30,45 o $\mathrm{C}$ en Alf-2, mientras que la mínima promedio fue de 27,8 oC en el manantial Alfonsina. El valor mínimo y máximo extremo fue de $20,1{ }^{\circ} \mathrm{C}$ y $38{ }^{\circ} \mathrm{C}$ en Alf-2.

Los niveles promedio de oxígeno oscilaron entre $3,88 \mathrm{mg} / \mathrm{L}(E E= \pm 0,56)$ en el manantial Alfonsina, hasta $5,9 \mathrm{mg} / \mathrm{L}$ $(E E= \pm 0,55)$ en la ciénaga media Oeste. $E I$ $\mathrm{pH}$ promedio osciló entre 7,40 $(E E= \pm 0,25)$ en el manantial Alfonsina y $7,99(E E= \pm 0,30)$ en Alf-2. Los valores promedio de redox fluctuaron entre -114 $\mathrm{mV}(\mathrm{EE}= \pm 37,5)$ en Alf-2 y $-46,1 \mathrm{mV}$ $(E E= \pm 33,8)$ en el manantial Alfonsina. Los valores extremos mínimo y máximo fueron de $-383 \mathrm{mV}$ y de $198 \mathrm{mV}$ en Alf-1.

Sólo se encontraron diferencias entre sitios en la salinidad superficial $\left(X^{2}=26,219, \quad p=0,001\right)$ e intersticial $\left(X^{2}=24,819, p=0,001\right)$, así como en la profundidad $\left(X^{2}=12,140, p=0,007\right)$. Por otra parte, se encontraron diferencias entre temporadas (secas e inundación) en la profundidad $\left(X^{2}=13,86, p=0,03\right)$ y oxígeno disuelto $\left(X^{2}=14,38, p=0,03\right)$ de la ciénaga media Oeste y en el oxígeno $\left(X^{2}=16,77, p=0,01\right)$ de la ciénaga media 
(a) Alfonsina, mangle rojo.

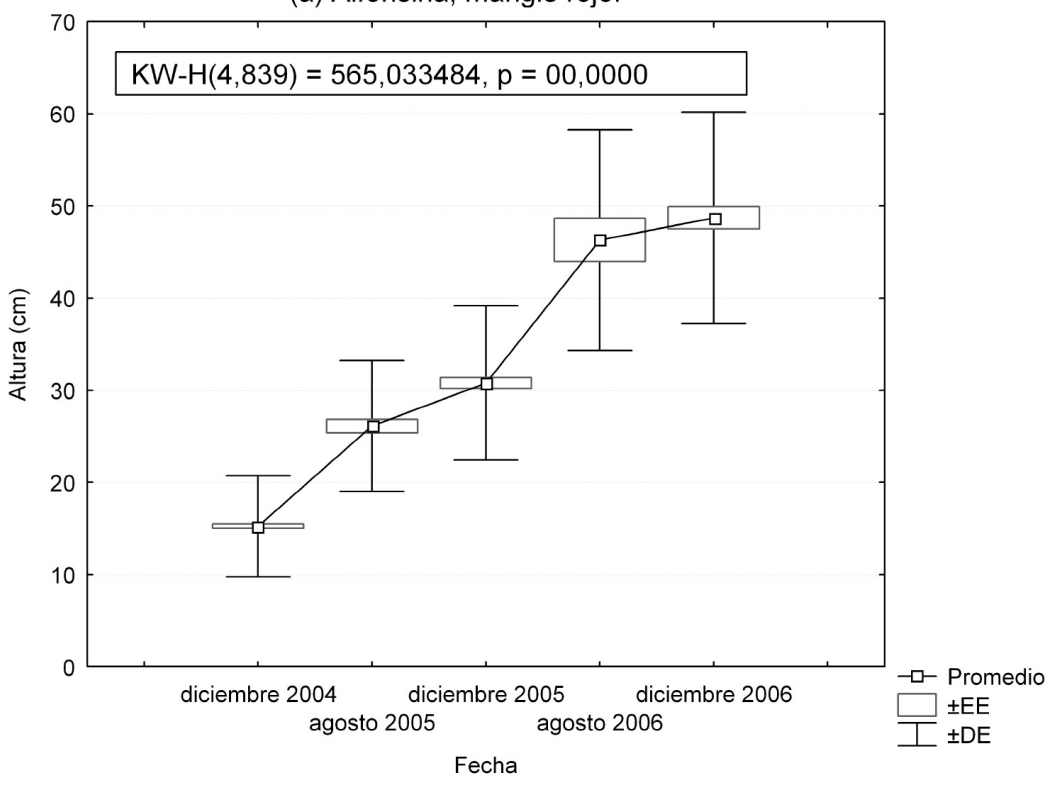

(b) Alfonsina, mangle negro.

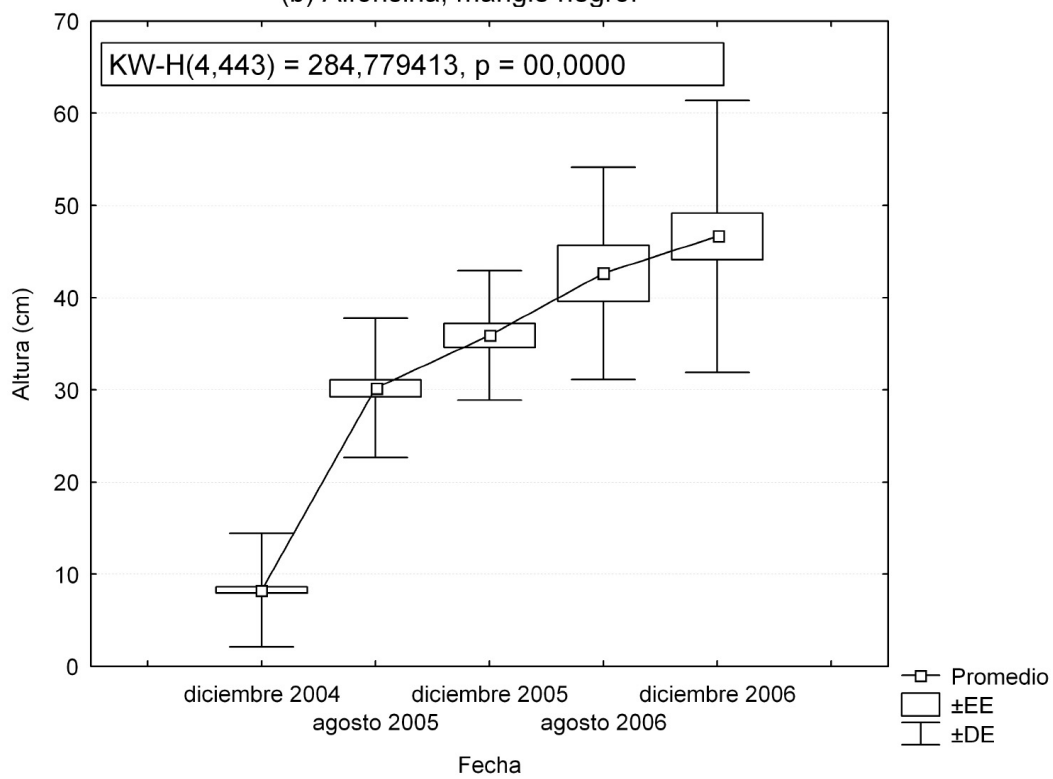

Figura 5. Crecimiento de las plántulas de (a) mangle rojo y (b) negro en Alfonsina. Se muestra el análisis de Kruskal-Wallis correspondiente. 
(a) Victoria, mangle rojo.

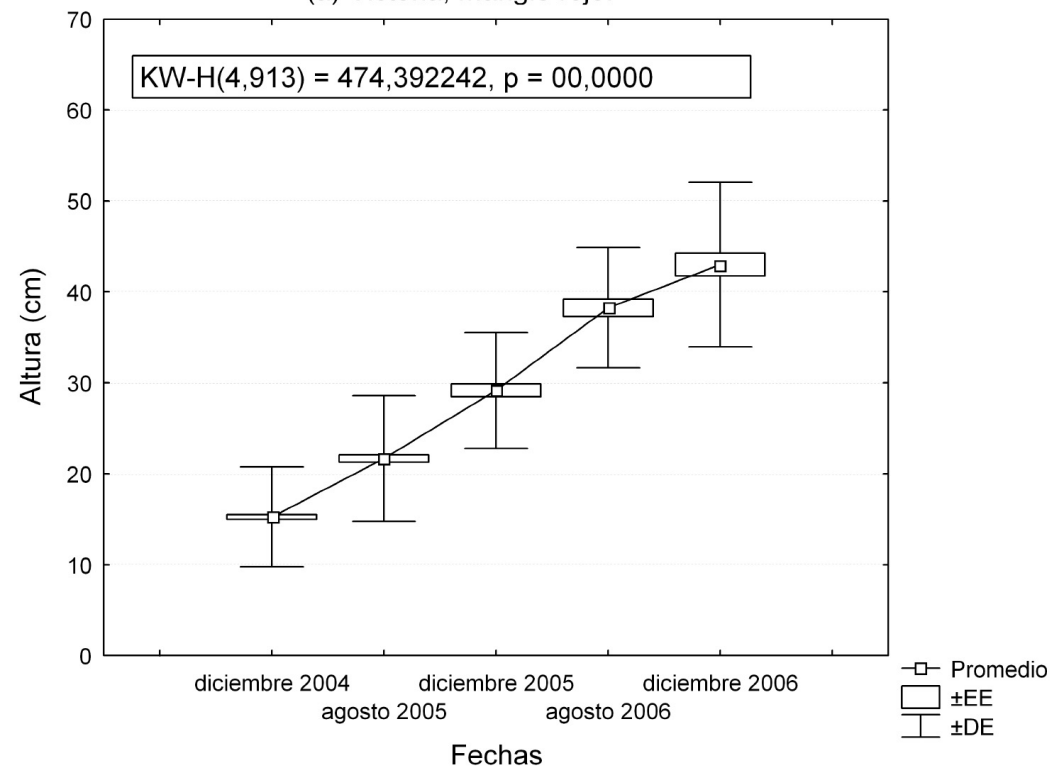

(b) Victoria, mangle negro.

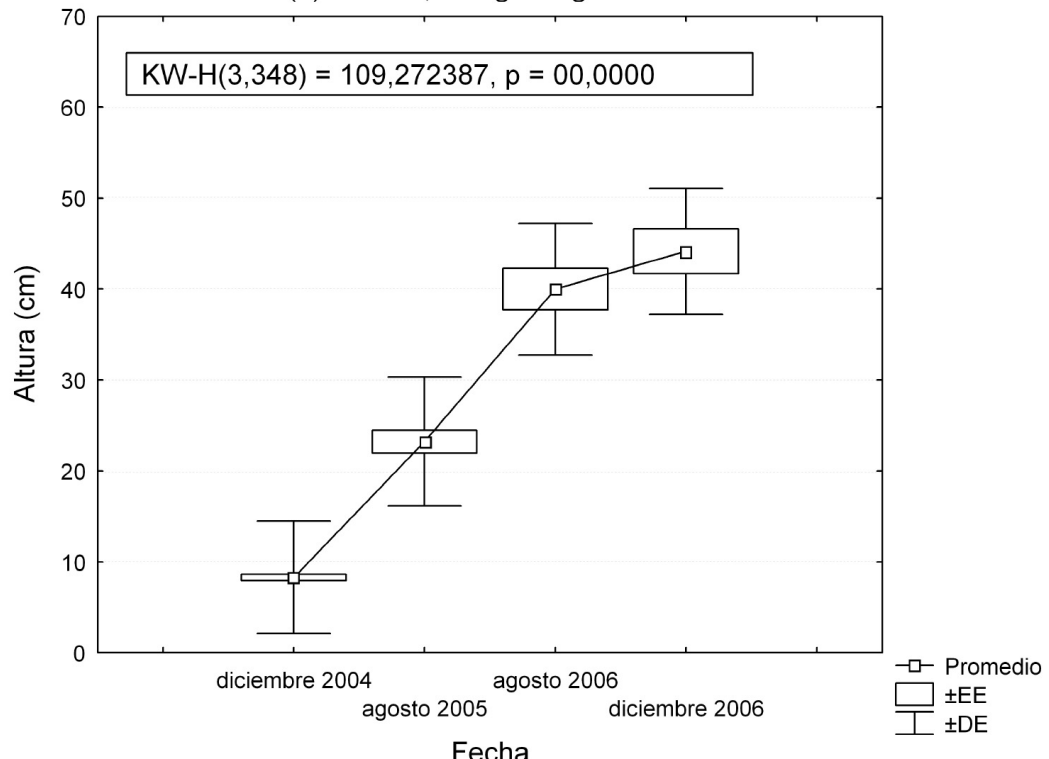

Figura 6. Crecimiento de las plántulas de (a) mangle rojo y (b) negro en Victoria. Se muestra el análisis de Kruskal-Wallis correspondiente. 
(a) Camas, mangle rojo

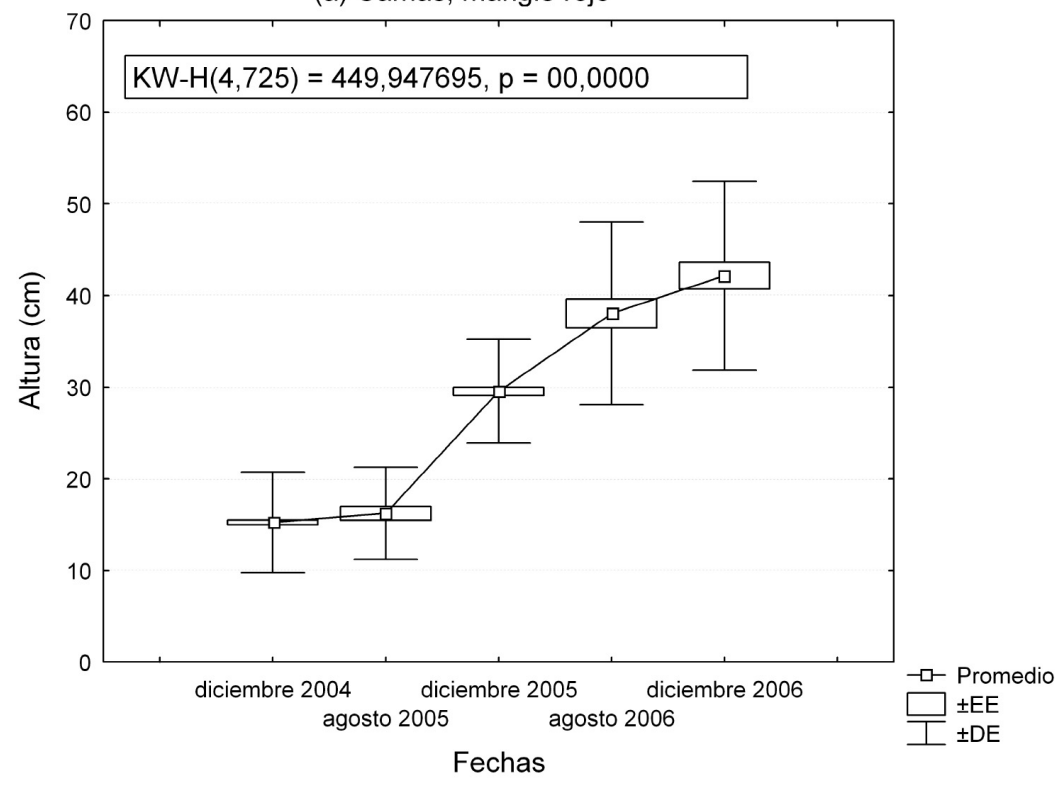

(b) Camas, mangle negro.

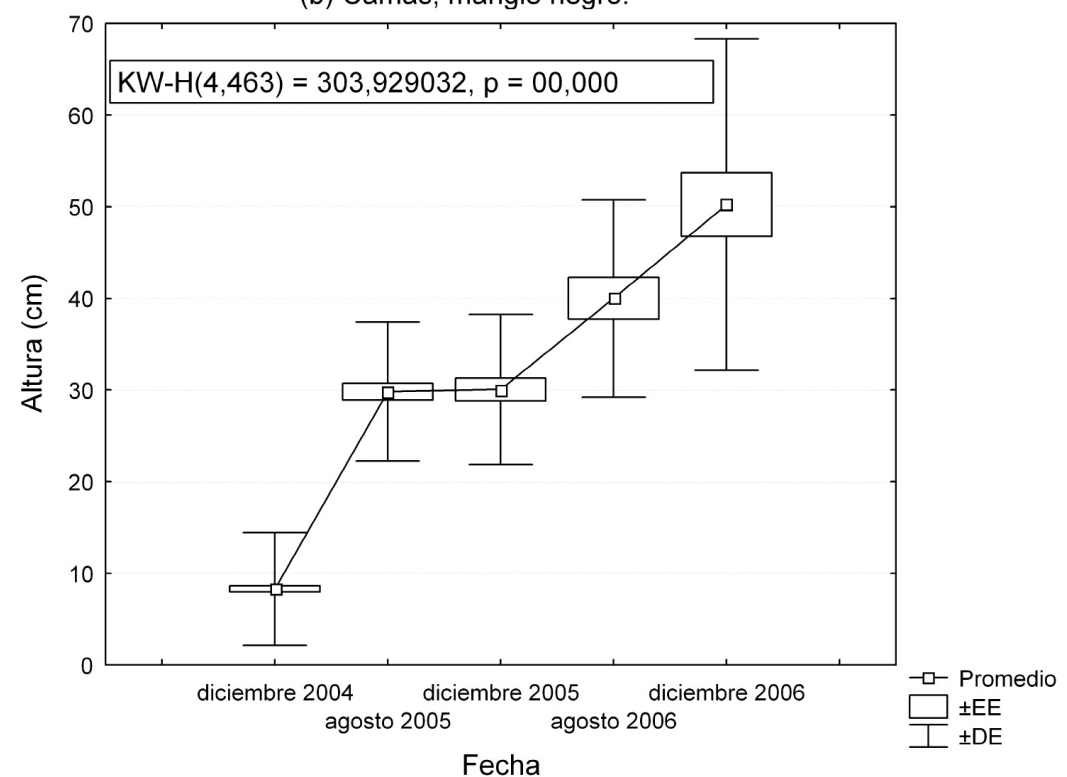

Figura 7. Crecimiento de las plántulas de (a) mangle rojo y (b) negro en las camas de El Cambio. Se muestra el análisis de Kruskal-Wallis correspondiente. 
(a) Canales, mangle rojo.

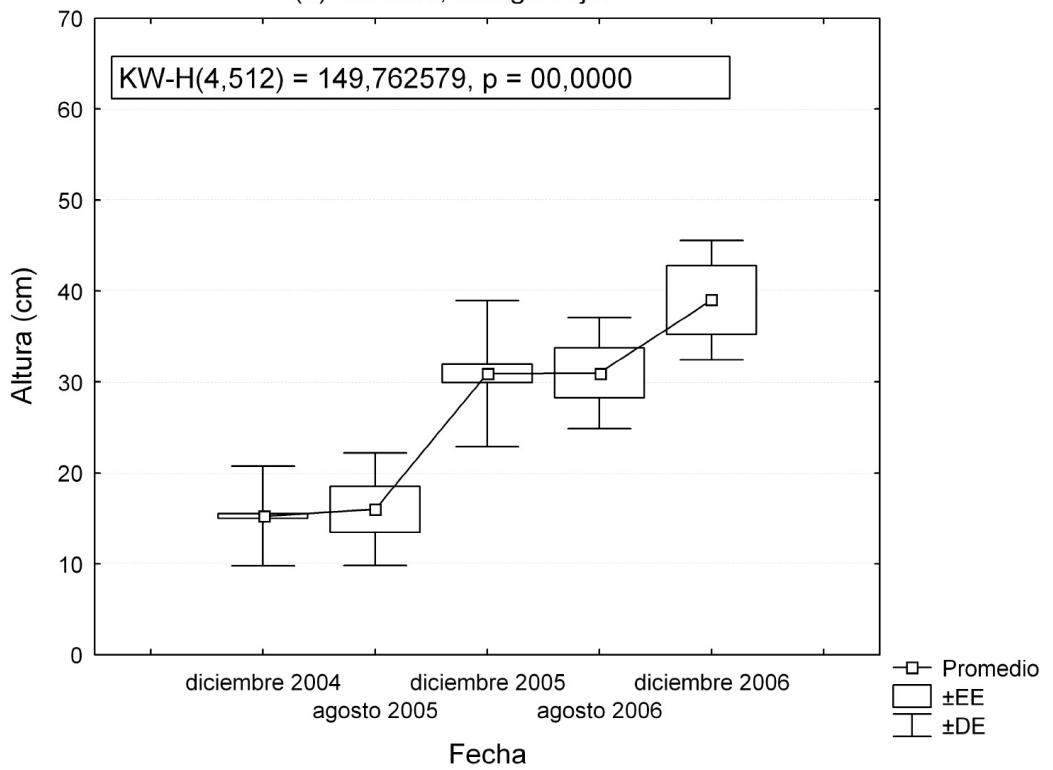

(b) Canales, mangle negro.

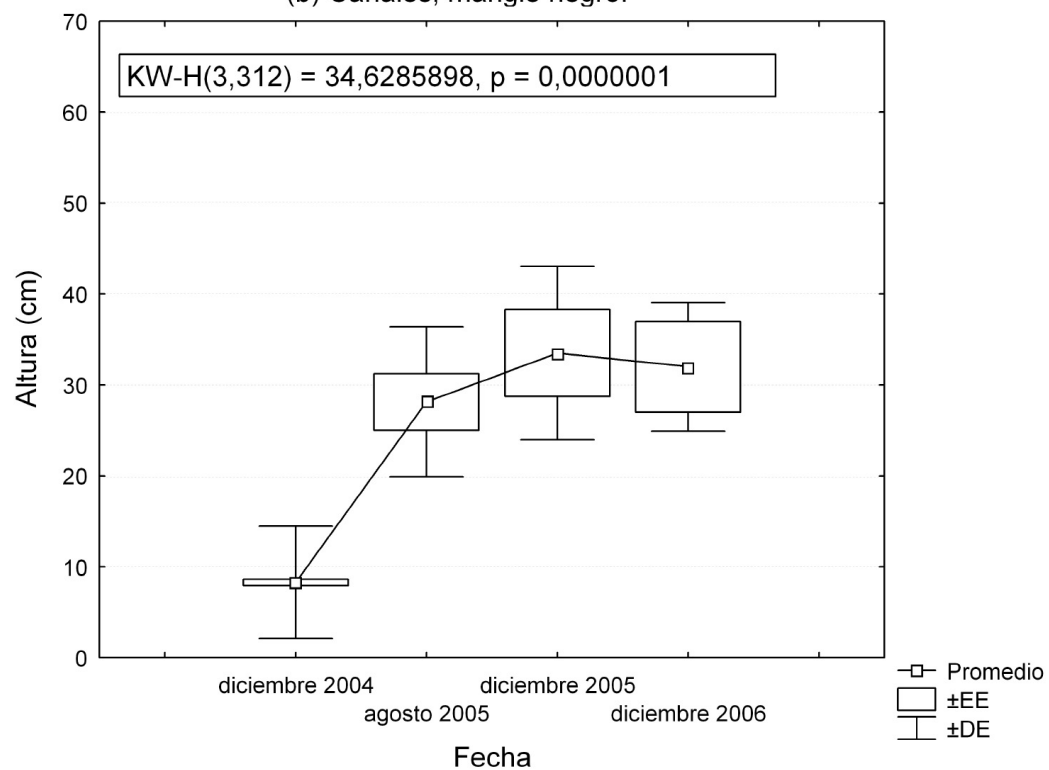

Figura 8. Crecimiento de las plántulas de (a) mangle rojo y (b) negro en los canales de El Cambio. Se muestra el análisis de Kruskal-Wallis correspondiente 
Este. En la ciénaga media Este, se registró un aumento en la salinidad promedio del agua de 33,4 ups, en la temporada seca de 2005, a 38,4 ups en la misma temporada de 2006, alcanzando un máximo de 43,1 ups en la temporada de inundación 2006-2007. Por el contrario, la profundidad promedio del agua bajó de $24,6 \mathrm{~cm}$ en la temporada seca de 2006, a 20,1 cm en la temporada de inundación 2006-2007.

\section{DISCUSIÓN}

Es ampliamente conocido que en la región del Caribe y Golfo de México, el mangle rojo ( $R$. mangle) puede crecer en aguas más profundas y con salinidades no mayores de 40 ups en el suelo, mientras que el mangle negro ( $A$. germinans) puede desarrollarse en suelos salinos de más de 50 ups y en zonas más someras con bajo hidroperiodo (McMillan, 1971; Lugo and Snedaker, 1974; Cintron et al., 1978; Jiménez, 1994; Rico-Gray y Palacios, 1996; Ramírez, 2005).

Lo anterior corresponde con nuestros resultados en Chabihau. Con las nuevas condiciones que se registran en la ciénaga, después de la construcción de puentes en la carretera costera, tales como un mayor reflujo de marea y una reducción de los niveles extremos de salinidad (Batllori y Febles, 2007), la profundidad y la salinidad del agua están controlando la sobrevivencia y el crecimiento de las plántulas de manglar. El mangle rojo presentó una mayor sobrevivencia bajo condiciones de mayor inundación (Victoria) y un mayor desarrollo con baja salinidad intersticial $(3,28$ ups \pm 0,32 ups; Alfonsina), mientras que el mangle negro presentó una mayor sobrevivencia en áreas menos inundadas y un mayor desarrollo con más alta salinidad (45,5 ups $\pm 0,50$ ups; El Cambio).
La construcción de camas de sedimento para controlar el grado de inundación, demostró ser una técnica útil para favorecer la sobrevivencia de mangle negro. En 2005, Novelo reportó una sobrevivencia de mangle rojo de $38 \%$ en siete meses, utilizando el método de camas de sedimento y el desazolve de manantiales en la ciénaga de Progreso, al noroeste de Chabihau. Sin embargo, el autor menciona que la baja sobrevivencia fue resultado de utilizar mangle rojo en condiciones de alta salinidad (de 54 ups a 72 ups después del acondicionamiento). Algunos experimentos de crecimiento de plántulas (desde algunas semanas hasta varios meses de edad) indican que el óptimo crecimiento de varias especies se alcanza a salinidades bastante más bajas que el agua de mar (<35 ups; Medina, 1998).

En Alfonsina, con la menor salinidad, las plantas tuvieron mayor crecimiento (mayor altura, número de ramas y raíces adventicias), incluso con presencia de flores y frutos al finalizar los dos primeros años de desarrollo. Por el contrario, la zona de Canales, con la mayor salinidad de todos los sitios, presentó el menor desarrollo de mangle rojo. El mangle negro presentó un mayor desarrollo (altura, número de hojas y de ramas) en Alfonsina, El Cambio y Victoria, respectivamente. Nuevamente, en Canales se observó el menor desarrollo de mangle negro, donde por cierto, se registró la más alta salinidad intersticial $(\mathrm{CMO}=46$ ups). Debido a las características semiáridas de la región, además del escaso flujo de agua dulce a través de manantiales y la poca profundidad del suelo, la altura máxima registrada en Alfonsina fue mucho menor a la registrada en la costa de Chiapas, donde Tovilla et al. (2004) registran una altura de $97,5 \mathrm{~cm}$ y 156,2 $\mathrm{cm}$ para el mangle rojo de vivero y de la siembra directa respectivamente, en menos de siete meses de crecimiento. 
Después de la construcción del bordo en el área de reforestación de Alfonsina, se registró una disminución de la salinidad del agua de 42,1 ups a 14 ups (15 días) y 1,5 ups (30 días), mientras que en la ciénaga adyacente la salinidad se mantuvo entre 40 ups y 45 ups (eneromarzo de 2004). La salinidad del agua superficial en la zona de Victoria, siembra directa, Canales y El Cambio, presentó características marinas (promedio de 32,7 ups a 39,3 ups) debido a la influencia diaria del reflujo de agua marina, mientras que en el área de Alfonsina fue menor (15,7 ups) y significativamente diferente a los otros sitios, debido a la influencia del manantial y el bordo construido.

En 1995, Febles y Batllori registraron un aumento promedio de $32 \mathrm{~cm}$ en la elevación del nivel de agua de un manantial, después del desazolve, aumentando el flujo de agua más dulce hacia la ciénaga. Lo anterior demuestra la importancia del desazolve de manantiales en áreas costeras cársticas, semiáridas, donde la descarga de agua subterránea controla el gradiente de salinidad. El proceso de dispersión y establecimiento de los propágulos de mangle, depende de varios factores como la distribución y la densidad de árboles maduros, la producción de propágulos, además del nivel de inundación, la salinidad, la temperatura del agua y el flujo de marea (Rabinowitz, 1978a; McKee, 1995; Ramírez, 2005). En Alfonsina y Victoria (lado Este de la ciénaga) se registró una mayor repoblación natural de mangle negro que en El Cambio y Canales (lado Oeste), debido posiblemente a la mayor densidad y desarrollo de la vegetación en las áreas cercanas, además del mayor reflujo de marea. Es posible que la carretera sea un obstáculo que afecta la dispersión de propágulos entre ambos lados de la carretera. En general, la menor profundidad y mayor salinidad del agua registrados en la ciénaga en 2006, asociado a un año menos lluvioso, pueden estar relacionados con la mayor incorporación natural de mangle negro observado en ese periodo. Elster et al. (1999) mencionan para la Ciénaga Grande de Santa Marta, Colombia, que el crecimiento de plántulas está controlado principalmente por los cambios en la salinidad, mientras que la inundación estacional regula la mortalidad. Los mismos autores mencionan una mortalidad de $100 \%$ de mangle negro cuando la inundación alcanzó $30 \mathrm{~cm}$ y cubrió completamente la parte aérea de las plantas. En áreas desprovistas de vegetación la regeneración es afectada por la salinidad extremadamente alta, temperaturas altas y desecación de la superficie del suelo. Las condiciones físicas extremas pueden ser críticas para la sobrevivencia de plántulas de manglar (Rabinowitz, 1978b; Reyes y Tovilla, 2002), de forma que la mortalidad es alta durante la fase de establecimiento, cuando las raíces están desarrollándose (Elster et al., 1999; Elster, 2000).

Tovilla-Hernández y Orihuela (2002) observaron el desarrollo de propágulos de $R$. mangle en condiciones de sombra (50\% de iluminación) e iluminación directa (100\%), encontrando durante el primer año que la mayor mortalidad se registró al sol, ya que $49,5 \%$ perecieron; en comparación con $38,5 \%$ de aquellas desarrolladas a la sombra. Lo anterior coincide con las observaciones de Febles et al. (2007), quienes registran una mayor sobrevivencia y desarrollo de raíces primarias en propágulos de mangle rojo provenientes de Chabihau, bajo condiciones de agua dulce ( $<5$ ups) y sombra, que en condiciones de agua más salada (>35 ups) e insolación directa. Es posible que existan asociaciones positivas entre individuos, de manera que el establecimiento podría ser un factor importante en la formación de parches de vegetación. 
En nuestra área de estudio, debido al mayor reflujo de marea después de la construcción de los puentes, la temperatura máxima promedio del agua fue de $30,4 \circ \mathrm{C}$, con extremos de $20,1{ }^{\circ} \mathrm{C}$ a $38{ }^{\circ} \mathrm{C}$ en Alf 2 , sin existir diferencias significativas entre sitios. En un ciclo de $24 \mathrm{~h}$ realizado del 2 al 3 de agosto de 2005, se registró una temperatura de $34{ }^{\circ} \mathrm{C}$ y 35,5 o $C$ en la ciénaga media Este (CME), a las 13 y $16 \mathrm{~h}$, respectivamente. En este sentido, Lugo y Snedaker (1974) y Clough et al. (1982), mencionan que las temperaturas óptimas para el adecuado desarrollo de los procesos fotosintéticos de los manglares se registran alrededor de los $35 \circ$ C.

La mortalidad al sol está también relacionada con el flujo de mareas a lo largo del año (Tovilla-Hernández y Orihuela, 2002). Ellison y Farnsworth (1993) observaron en Belice que $R$. mangle presentó mayor sobrevivencia relacionada con una mayor influencia de las mareas, independientemente de si las plántulas estuvieran bajo el dosel del bosque o al sol. McKee (1995c) indica que el proceso de arraigo temprano de los propágulos está más relacionado con la disponibilidad de nutrientes en el medio que con la luz. En Chabihau, Teutli (2004) encontró en la ciénaga valores medios anuales de nitrógeno inorgánico disuelto (NID) de 29,3 $\mu \mathrm{mol}$ (lado Oeste) y $25,8 \mu \mathrm{mol}$ (lado Este), así como 0,87 $\mu \mathrm{mol}$ (lado Oeste) y 1,72 $\mu \mathrm{mol}$ (lado Este) de fósforo, a ambos lados de la carretera. Estos valores fueron superiores a los encontrados en otras áreas de la costa de Yucatán con un mayor desarrollo del manglar, pero la disponibilidad puede estar controlada por otros factores como el oxígeno, el $\mathrm{pH}$ y el potencial redox. En el área de Chabihau estos parámetros no presentaron diferencias significativas entre los sitios.

De acuerdo con Elster (2000) y Reyes y Tovilla (2002), la siembra directa de propágulos de mangle rojo puede ser un método más económico, además de que se puede cubrir una superficie mayor de reforestación; sin embargo, es posible obtener una mayor sobrevivencia con plántulas de vivero dependiendo de las condiciones de cada sitio (Tovilla et al., 2004). La sobrevivencia de mangle rojo en Victoria (producidas en vivero) fue el doble de la registrada en la siembra directa, durante el primer año de crecimiento; no obstante, las tasas de crecimiento fueron similares. Es posible que la mortalidad registrada durante el primer año en las plántulas de mangle, esté relacionada con problemas de manejo en el vivero, como la falta de un cambio gradual en la salinidad del agua de riego y en las condiciones de insolación.

Para desarrollar métodos de restauración de manglar en ciénagas costeras semiáridas, es necesario conocer los factores que afectan los patrones de producción, dispersión y establecimiento de propágulos. Para el diseño de programas de reforestación se debe contar con un monitoreo de parámetros hidrológicos superficiales y del suelo (particularmente nivel de inundación y salinidad) que permita la selección de las especies y los métodos adecuados para asegurar mayor sobrevivencia y mejor desarrollo de las plantas.

\section{CONCLUSIONES}

Con las nuevas condiciones que se registran en la ciénaga de Chabihau, después de la construcción de puentes en la carretera costera, tales como un mayor reflujo de marea y una reducción de los niveles extremos de salinidad, la sobrevivencia de plántulas de manglar está regulada por el nivel de inundación, mientras que la salinidad del agua controla su crecimiento. 
La construcción de camas de sedimento para controlar el grado de inundación, demostró ser una técnica útil para favorecer la sobrevivencia de mangle negro, mientras que el desazolve de manantiales y la disminución de la salinidad del agua, aseguran un mejor desarrollo de las plántulas de ambas especies.

\section{AGRADECIMIENTOS}

A las mujeres del grupo "Flor de Mangle" de Chabihau, por su entusiasmo durante el desarrollo del proyecto. El Consejo para la Conservación de los Humedales de Norteamérica (NAWCC) financió las obras de restauración en Chabihau, permitiendo la realización de este estudio. Petróleos Mexicanos (PEMEX) apoyó con combustible para el trabajo de campo. El Dr. Cristian Tovilla y dos revisores anónimos enriquecieron con sus comentarios el manuscrito. Elisa Cuevas, Héctor Rodríguez y Humberto Medina participaron en diferentes momentos del trabajo de campo.

\section{REFERENCIAS}

Batllori-Sampedro, E., J. L. Febles-Patrón y J. Díaz-Sosa. 1999. Landscape change in Yucatan's northwest coastal wetlands (1948-1991). Human Ecology Review 6(1): 8-20.

Batllori, E., J. González, J. Díaz y J. L. Febles. 2006. Caracterización hidrológica de la región costera noroccidental del estado de Yucatán, México. Investigaciones Geográficas, Boletín del Instituto de Geografía, UNAM, 59: 74-92.

Batllori, E. y J. L. Febles. 2007a. Cambio en la salinidad de la laguna costera de Chabihau, Yucatán, México. Efecto del huracán Isidoro y la cons- trucción de puentes en la duna costera. Ingeniería Hidráulica en México XXII (3): 61-69.

Batllori, E. y J. L. Febles. 2007b. Changes in the hydrological characteristics of Chabihau coastal wetlands, Yucatan, Mexico, associated with hurricane Isidore impact. Indian Journal of Marine Sciences 36(3):183-192.

Cintrón, G., A. E. Lugo, D. J. Pool and Morris, G. 1978. Mangroves of arid environments in Puerto Rico and adjacent islands. Biotropica 10: 110-121.

Clough, B. F., T. J. Andrews and I. R. Cowan. 1982. Physiological processes in mangroves. En: B. Clough (ed.). Mangrove Ecosystems in Australia: Structures, Function and Management, ANU Press, Canberra. p: 193-210.

CONAGUA, 2006. Información climatológica de Telchac Puerto, Yucatán. Gerencia Regional de la Comisión Nacional del Agua.

Craighead, F. C. 1971. The tree of South Florida. Volume 1. The natural environments and their succession. University of Miami Press. Coral Gables, FL.

Duch, J. 1988. La Conformación Territorial del Estado de Yucatán. Los Componentes del Medio Físico. Universidad Autónoma de Chapingo. Centro Regional de la Península de Yucatán, México, 427 p.

Ellison, A., M. y E. J. Farnsworth. 1993. Seedling survivorship growth and response to disturbance in Belizean mangroves. American Journal of Botany 80: 1137-1145. 
Elster, C., L. Perdomo-Trujillo y M. L. Schnetter. 1999. Impact of ecological factors on the regeneration of mangroves in the Ciénaga Grande de Santa Marta, Colombia. Hydrobiologica 413: 35-46.

Elster, C. 2000. Reasons for reforestation success and failure with three mangrove species in Colombia. Forest Ecology and Management 131: 201-214.

Febles-Patrón, J. L. y E. Batllori. 1995. Fluctuación diurna del nivel hidrostático en petenes de la cuenca costera noroccidental del estado de Yucatán: efecto del desazolve y la canalización de manantiales. Ingeniería Hidráulica en México X(2): 5-19.

Febles-Patrón, J. L, J. Novelo y E. Batllori. 2007. Efecto de factores abióticos en el desarrollo de raíces primarias, crecimiento y supervivencia de propágulos de Rhizophora mangle L. Revista Madera y Bosques 13(2): 15-27.

García, E. 1988. Modificaciones al sistema climático de Köppen. México. D. F., 2a . ed., 243 p.

Jiménez, J. A. 1994. Los mangles del Pacífico Centroamericano. Universidad Nacional, Instituto Nacional de Biodiversidad. Costa Rica, 336 p.

Lugo, A. E. and S.C. Snedaker. 1974. The ecology of mangroves. Annual Review of Ecology and Systematics 5: 39-64.

McKee, K. L. 1993. Soil physicochemical patterns and mangrove species distribution-reciprocal effects? Journal of Ecology 81: 477-487.

McKee, K. L. 1995a. Seedling recruitment patterns in a Belizean mangrove forest: effects of establishment ability and physicochemical factors. Oecologia 101(4): 448-460.

McKee K. L. 1995b. Mangrove species distribution and propagules predation in Belize: an exception to the dominance-predation hypothesis. Biotropica 27(3): 334-345.

McKee K. L. 1995c. Interspecific variation in growth, biomass partitioning, and defensive characteristics of Neotropical mangrove seedlings: response to light and nutrient availability. American Journal of Botany 82 : 299-307.

McMillan, C. 1971. Environmental factors affecting seedling establishment of the black mangrove on the central Texas coast. Ecology 52: 927-930.

Medina, E. 1998. Mangrove physiology: the challenge of salt, heat, and light stress under recurrent flooding. IV Simposio de Ecosistemas Brasileiros, Academia de Ciencias de Sao Paulo, Brasil V: 25-56.

Novelo, J. 2005. Evaluación del método "camas de sedimento" para la reforestación de manglar (Rhizophora mangle L.) en la ciénaga de Progreso, Yucatán, México. Tesis de Licenciatura. Universidad Autónoma de Yucatán, México, 85 .

Perry, E., J. Swift, J. Gamboa, A. Reeve, R. Sanborn, L. Marin and M. Villasuso. (1989). Geologic and Environmental Aspects of Surface Cementation, North Coast, Yucatan, Mexico. Geology 17: 818-821.

Rabinowitz, D. 1978a. Dispersal Properties of Mangrove Propagules. Biotropica 10(1): 47-57. 
Rabinowitz, D. 1978b. Early growth of mangrove seedlings in Panamá, and an hypothesis concerning the relationship of dispersal and zonation. Journal of Biogeography 5: 113-133.

Ramírez, L. 2005. Factores que afectan la propagación y establecimiento de Avicennia germinans $\mathrm{L}$. en ambientes degradados de regiones semiáridas subtropicales. Tesis de Maestría en Ciencias Marinas. Universidad de Puerto Rico, 108 p.

Reyes-Chargoy, M. A. y C. TovillaHernández. 2002. Restauración de áreas alteradas de manglar con Rhizophora mangle en la Costa de Chiapas. Maderas y Bosques, número especial 8: 103-114.

Rico-Gray, V. y M. Palacios. 1996. Salinidad y el nivel del agua como factores en la distribución de la vegetación en la ciénaga del NW de Campeche. México. Acta Botánica Mexicana 34: 53-61.

Teutli, H. C. 2004. Estimación del éxito de la restauración hidrológica de zonas de manglar en el norte de Yucatán,
México. Tesis de Licenciatura. Escuela de Biología. Universidad Autónoma de Puebla, 51 p.

Tomlinson, P. B. 1994. The botany of mangroves. Harvard University. Harvard Forest. Petersham, Mass, $419 \mathrm{p}$.

Tovilla-Hernández, C. y D. E. Orihuela. 2002. Floración, establecimiento de propágulos y supervivencia de Rhizophora mangle L. en el manglar de barra de Tecoanapa, Guerrero, México. Madera y Bosques, número especial 8: 89-102.

Tovilla, C., A. Román, G. Simuta y R. Linares. 2004. Recuperación del manglar en la Barra del Río Cahoacán, en la costa de Chiapas. Madera y Bosques, número especial 2: 77-91.

Yáñez-Arancibia, A., R. Twilley y A. LaraDomínguez. 1998. Los ecosistemas de manglar frente al cambio climático global. Madera y Bosques 4(2): 3-19. reforestación de mangle en una ciénaga costera semiárida de Yucatán, México. Madera y Bosques 15(3): $65-86$ 